Patient voices

\title{
Getting the COVID-19 vaccine as a transplant patient
}

\section{Michael Mittelman}

10.1136/bmjebm-2021-111740

Correspondence to:

Michael Mittelman, Philadelphia, Pennsylvania, USA; mikemitt@gmail.com

\section{Check for updates}

( $)$ Author(s) (or their employer(s)) 2021. No commercial re-use. See rights and permissions. Published by BMJ.

\section{To cite: Mittelman $M$.}

BMJ Evidence-Based

Medicine Epub ahead of

print: [please include Day

Month Year]. doi:10.1136/

bmjebm-2021-111740

\section{Getting the vaccine appointment}

'Yes! I am fully vaccinated', I said to myself. The thrill, the excitement, coupled with too many other emotions to describe came swelling over me. I was also all alone, waiting in a room with others who had recently received their first or second dose of a COVID-19 mRNA vaccine. I could sense a feeling of cautious joy in the room as I looked around at others also sitting with their masks on. At 39 years of age, I guessed that I was the youngest person in the room.

I felt 'chosen' and privileged to be among the first to be vaccinated. Several weeks before, when my doctor's office called me on a Saturday, I worried something was wrong. 'Your doctor has referred you for the vaccine', they said. 'We are beginning to offer them to our transplant patients. Would you like the vaccine?' Silence. Would I? Heck yes! My family and friends had been wondering when it might be my turn, when the city would begin to allow transplant recipients to have the vaccine. I responded, 'Yes! Tell me where and when, and I will be there!'

I have an underlying rare kidney disease, have had three kidney transplants (the current one from a living donor) and have a number of other chronic health conditions. The pandemic has been a challenge for everyone, but it has been particularly challenging for those living with underlying conditions and people with compromised immune systems like me.

\section{COVID-19 vaccines in people with transplants}

When it was announced that vaccines were going to be approved, the transplant community began talking about who should get the vaccine and when. As early as late December, with the Food and Drug Administration's emergency use authorisations for the Pfizer and Moderna vaccines, professional medical organisations, including the American Society of Transplantation (AST), recommended that transplant patients should receive the COVID-19 vaccine once it became available to them, while acknowledging the need for further research in transplant recipients. More data about the safety of the vaccines in transplant recipients are needed, but according to expert opinion, their mechanism of action does not suggest they are more likely to trigger rejection episodes or unexpected or more severe side effects. ${ }^{1-3}$

My transplant centre at the University of Pennsylvania also echoed the advice of the AST that transplant patients should get the COVID-19
mRNA vaccines. Despite these recommendations, many transplant patients remain apprehensive and still do not want the vaccine, fearing the risks of getting the vaccine might outweigh the benefits in preventing infection, a sentiment shared by many other rare disease and immunosuppressed patients. Happily, as more transplant patients went on to be vaccinated, early theoretical concerns of activation of the immune system by the vaccine, triggering episodes of rejection, have not been observed. ${ }^{4}$ However, questions still remain about the efficacy of the vaccine in immunosuppressed patients like me.

\section{COVID-19 antibody testing after the vaccine}

Clinical protocols are not the same in every transplant programme. My transplant centre tests for early signs of rejection in patients 1 month after the second dose of the vaccine by checking for donor specific antibodies (DSAs) and by monitoring the health of the transplant with regular blood tests. They want to check to see if an immune response triggered by the vaccine might also cause my body to be rejecting and/or recognising the transplanted kidney as a foreign body. This has become part of their clinical protocol for kidney, pancreas, kidney-pancreas, heart-kidney and a few other combinations of transplants that tests for DSAs. Thankfully, I got my tests done, and all was good with my kidney transplant.

Ongoing studies are evaluating whether solid organ transplant recipients respond to the Pfizer or Moderna vaccine. I tried to enrol in these studies, but was not selected. Results from the first studies in transplant patients after only one mRNA vaccine dose were published in JAMA in March, ${ }^{5}$ and the findings were not very encouraging, with only $17 \%$ of transplant patients studied developing antibodies after the first dose alone. The study, which suggested that transplant recipients should get COVID-19 antibody tests, subsequently gained media attention by numerous outlets, including one in my hometown of Philadelphia. ${ }^{6}$ My family and friends wondered if the vaccine worked on me and encouraged me to get my antibodies checked. I went into a sort of panic mode. I tried hard to get the spike protein antibody blood test that would distinguish vaccine response from true infection but was unable to find a doctor who would order it since routine antibody testing after vaccination is not currently recommended by the CDC or AST.

One month after my second dose, I found a local pharmacy that would offer me a rapid COVID-19 
antibody test. I waited for full 20 min with bated breath for the results. We look ... NOTHING! I said, 'What does that mean?'. The nurse said, 'Well, according to this test, you have no detectable levels of COVID-19 antibodies'. I responded, 'Does this mean the vaccine did not work?'. She would not answer that question. I called my family, and then I messaged my nephrologist and transplant surgeon. My transplant surgeon hopped on a Zoom chat with me on a Sunday and told me to calm down. He told me that we know transplant patients might have a suppressed response as they do to other vaccines, like the influenza vaccine, $a n d^{12}$ we do not know how accurate this rapid test was. I was left wondering, 'if it is not accurate, then why would be it be offered all over the country and why would the FDA allow it to be used?'

\section{A call for research, shared decision-making and empowering patients with knowledge}

As a transplant patient, will the vaccine work for me? The excitement that came with being vaccinated now turned to questions and more worries. Should physicians recommend antibody testing after vaccination for transplant patients? What does this mean not only for me, but for friends who have compromised immune systems due to cancer treatments or other autoimmune diseases? Am I allowed to resume activities that others are? Can I see friends who are vaccinated or have only one dose? More recent studies from around the globe support the earlier findings that response to vaccines is low in the immunosuppressed, but the studies had small numbers of patients. ${ }^{7-9}$ The second part of the Hopkins study showed slightly more promise after the second dose of the vaccine. ${ }^{4}$ We still need more clinical data, but how quickly will it arrive?

The low response rate to vaccines in transplant patients sparked debate among both patients and physicians about the risks and the benefits of reducing immunosuppression prior to vaccination to maximise immune response. I would not go this route even if it were to become a recommendation. The risk of rejection to my third kidney transplant would be too great for me. From what I understand, we still may have some protections through $\mathrm{T}$ cells and in our immune system's memory cells.

I see this as a call to research and a call to community empowerment. What is the purpose and role of antibody testing after vaccination? Are we testing for the purpose of research or new protocols? Are the expectations of someone in a trial different than for just me? Furthermore, patients may not get the right test, which could make them either worry more than necessary or have a false sense of security. If we are going to be doing antibody testing in retail stores, then we need to empower and educate patients about the limitations of these tests. We cannot interpret rapid tests the same as laboratory-based tests; this means the community practitioners in these retail settings and other care settings must be prepared to explain this to patients.

I view this as a call to clinicians, nurses, social workers, coordinators and other health professionals to have an honest discussion with your patients. Discuss the options and reach a shared decision about the best next step-a research trial, a blood test, another form of immune response study or no further action. Whatever that decision is, it is meaningful to that patient, their family and the community. It is time that we do our best to remove some of the uncertainties in our lives. We can only accomplish this with mutual respect and a shared sense of trust.

Correction notice This article has been corrected since it appeared Online First. Minor text change, repitition of sentence.

Acknowledgements I would like to thank Amy Price and Ken Sutha for their input to the manuscript.

Contributors MM devised and wrote the article.

Funding The authors have not declared a specific grant for this research from any funding agency in the public, commercial or not-for-profit sectors.

Competing interests $\mathrm{MM}$ is a transplant recipient and rare disease patient advocate. He works in cybersecurity, is a former Patient Editor at the BMJ, a global PCORI ambassador and is the Chairman of the American Living Organ Donor Fund.

Patient consent for publication Not required.

Provenance and peer review Not commissioned; internally peer reviewed.

\section{References}

1 myast.org. COVID-19 vaccine FAQ sheet. American Society of transplantation, 2021. Available: https://www.myast.org/sites/default/ files/2021\%2003\%2018\%20COVID19\%20VACCINE\%20FAQS_update.pdf [Accessed 06 May 2021].

2 myast.org. COVID-19 vaccine FAQ sheet. American Society of transplantation, 2020. Available: https://www.myast.org/sites/default/files/ 2020\%2012\%2024\%20COVID19\%20VACCINE\%20FAQS_CLEAN-v2.pdf [Accessed 06 May 2021].

3 American Society of Transplant Surgeons. Transplant capacity and early vaccine recommendations. American Society of transplant surgeons, 2020. Available: https://asts.org/advocacy/covid-19-resources/asts-covid-19strike-force/transplant-capacity-in-the-covid-19-era [Accessed 06 May 2021].

4 Boyarsky BJ, Ou MT, Greenberg RS, et al. Safety of the first dose of SARS$\mathrm{CoV}-2$ vaccination in solid organ transplant recipients. Transplantation 2021;105:e56-7.

5 Boyarsky BJ, Werbel WA, Avery RK, et al. Immunogenicity of a single dose of SARS-CoV-2 messenger RNA vaccine in solid organ transplant recipients. JAMA 2021;325:1784-6.

6 McCullough M. COVID-19 vaccines don't protect most organ-transplant patients, study finds. The Philadelphia Inquirer, 2021. Available: https:// www.inquirer.com/health/coronavirus/covid-19-vaccination-may-beineffective-for-immunosupressed-patients-20210317.html [Accessed 06 May 2021].

7 Husain SA, Tsapepas D, Paget KF, et al. Post-vaccine anti-SARS-CoV-2 spike protein antibody development in kidney transplants recipients. Kidney Int Rep 2021. doi:10.1016/j.ekir.2021.04.017. [Epub ahead of print: 23 Apr 2021].

8 Marinaki S, Adamopoulos S, Degiannis D, et al. Immunogenicity of SARS-CoV-2 BNT162b2 vaccine in solid organ transplant recipients. Am J Transplant 2021. doi:10.1111/ajt.16607. [Epub ahead of print: 17 Apr 2021].

9 Grupper A, Rabinowich L, Schwartz D, et al. Reduced humoral response to mRNA SARS-CoV-2 BNT162b2 vaccine in kidney transplant recipients without prior exposure to the virus. Am J Transplant 2021. doi:10.1111/ ajt.16615. [Epub ahead of print: 18 Apr 2021]. 